\title{
EGU2020-20279
}

https://doi.org/10.5194/egusphere-egu2020-20279

EGU General Assembly 2020

(c) Author(s) 2021. This work is distributed under

the Creative Commons Attribution 4.0 License.

\section{Detection of sulphuric life in Mars analogue material using a miniature LIMS system}

\author{
Andreas Riedo ${ }^{1}$, Valentine Grimaudo ${ }^{2}$, Joost W. Aerts ${ }^{3}$, Alena Cedeño López ${ }^{4}$, Marek Tulej ${ }^{2}$, Pascale \\ Ehrenfreund ${ }^{5,6}$, and Peter Wurz ${ }^{2}$ \\ ${ }^{1}$ Department of Physics, Free University of Berlin, Berlin, Germany (andreas.riedo@fu-berlin.de) \\ ${ }^{2}$ Physics Institute, University of Bern, Bern, Switzerland \\ ${ }^{3}$ Faculty of Earth and Life Sciences, VU University Amsterdam, Amsterdam, The Netherlands \\ ${ }^{4}$ Department of Chemistry and Biochemistry, University of Bern, Bern, Switzerland \\ ${ }^{5}$ Leiden Observatory, Leiden University, Leiden, The Netherlands \\ ${ }^{6}$ Space Policy Institute, George Washington University, Washington DC, USA
}

In situ identification of life signatures on Solar System bodies other than Earth is extremely challenging and demands for sophisticated and sensitive instrumentation for their detection. Life signatures can be grouped into six different categories, ranging from biomolecules (e.g., lipids and amino acids), to microstructures (such as microfossils) [1]. Sulphur fractionated element isotopes belong to another important category and are of high interest to current astrobiology. In this contribution, we report on a novel measurement protocol, which is dedicated to measure accurately fractionated sulphur isotopes in different Mars analogue materials with an accuracy at the ${ }^{34} \delta$ level using our miniature and sensitive Laser Ablation Ionisation Mass Spectrometer (LIMS) that was designed for space exploration missions.

The applied LIMS instrument in this study consists of a miniature reflectron-type time-of-flight mass analyser (160 $\mathrm{mm} \times \varnothing 60 \mathrm{~mm})$ and a femtosecond laser system $(\lambda=775 \mathrm{~nm}, \tau \sim 190 \mathrm{fs})$ used as ablation and ionization source [2-3]. By means of irradiance studies performed on the Mars analogues, optimal measurement conditions could be elaborated, which allowed to measure sulphur fractionation with an accuracy at the ${ }^{34} \delta$ level.

All measurements presented here were conducted on five very different Mars analogues that were collected at different extreme field sites on Earth, including Rio Tinto in Spain and Movile and Sulphur caves in Romania. The analogues differ strongly in their total sulphur weight abundance, which range from $\sim 5$ to $\sim 95 \%$, and in their fractionation degree of sulphur $\left({ }^{34} \delta\right.$ from about +8 to -7). In comparison to the state-of-the-art sulphur isotope measurements the LIMS measurements showed an accuracy of $\sim 1.5^{34} \delta$. The measurement protocol is simple and sufficiently accurate for in situ application. It will provide valuable information of e.g., geochemical processes occurred on Solar System body surfaces, and will enable the identification of sulphuric-based life in case the fractionation is above fractionation induced by geochemical processes.

References 
1) E. Hays, H.V. Graham, D.J. Des Marais, E.M. Hausrath, B. Horgan, T.M. McCollom, M. Niki Parenteau, S.L. Potter-McIntyre, A.J. Williams and K.L. Lynch, "Biosignature Preservation and Detection in Mars Analog Environments", Astrobiol., 17, 2017, 363 - 400.

2) Riedo, M. Neuland, S. Meyer, M. Tulej and P. Wurz, "Coupling of LMS with a fs-laser ablation ion source: elemental and isotope composition measurements", J. Anal. At. Spectrom., 28, 2013, 1256 $-1269$.

3) Tulej, A. Neubeck, M. Ivarsson, A. Riedo, M.B. Neuland, S. Meyer and P. Wurz, "Chemical composition of micrometer-sized filaments in an aragonite host by a miniature laser ablation/ionization mass spectrometer", Astrobiol., 15, 2015, 669 - 682. 